\title{
Automated detection of sleep disordered breathing using a nasal pressure monitoring device
}

\author{
Sukhdev S. Grover • Stephen D. Pittman
}

Received: 1 November 2007 /Revised: 18 February 2008 / Accepted: 20 February 2008 /Published online: 27 March 2008

(C) Springer-Verlag 2008

\begin{abstract}
To assess the accuracy of a single channel portable monitoring device (RUSleeping ${ }^{\text {TM }}$ RTS, Respironics, Murrysville, PA) that measures nasal pressure (a surrogate for airflow) to detect sleep disordered breathing (SDB). Twenty-five adult patients referred to a community sleep laboratory with suspected obstructive sleep apnea (OSA) participated in this investigation. The portable monitoring device was used in the sleep laboratory to acquire data concurrently with a standard multi-channel polysomnogram (PSG) to assess SDB. Respiratory events were scored manually on the PSG using standard criteria for clinical research to quantify an apnea-hypopnea index (AHI) based on events during sleep. The portable monitoring device automatically calculated an unedited respiratory event index (REI) based on recording time. These data were then compared using the Pearson product-moment correlation coefficient, Bland-Altman analysis, receiver operating characteristic (ROC) curves, and likelihood ratios. All 25 subjects completed the study. Mean age of subjects was $42.4 \pm 12.9$ years and mean body mass index was $31.0 \pm$
\end{abstract}

This study was performed at the Sleep Center of Greater Pittsburgh Sleep Laboratory.

\section{S. S. Grover}

Sleep Center of Greater Pittsburgh,

Pittsburgh, PA, USA

S. D. Pittman

Division of Sleep Medicine, Brigham and Womens Hospital,

Boston, MA, USA

S. D. Pittman $(\bowtie)$

Sleep and Home Respiratory Group, Respironics, Inc.,

1505 Commonwealth Avenue, Suite 420,

Boston, MA 02135, USA

e-mail: stephen.pittman@respironics.com
$7.4 \mathrm{~kg} \mathrm{~m}^{-2}$. There was good agreement between the REI and the AHI $(R=0.77, p<0.001$, mean difference 2.6 events per hour [2 SD: 39.8 ] using a Bland-Altman plot). The area under the ROC curve for detecting SDB (PSG AHI greater than or equal to five events per hour) with the REI was 0.94 (95\% CI $0.84-1.0)$. For an REI $>11.9$ events per hour, the sensitivity was 0.89 (95\% CI $0.65-0.99)$ and the specificity was 0.86 (95\% CI $0.42-1.0)$ with a likelihood ratio of 6.2 for a positive test $(\mathrm{LR}+)$ and 0.13 for a negative test ( $\left.\mathrm{LR}^{-}\right)$. Similar results were observed for detecting moderatesevere SDB (PSG AHI $\geq 15$ events $\mathrm{h}^{-1}$ ) using REI $>15.2$ events $\mathrm{h}^{-1}$. In a population of subjects with suspected OSA, this portable monitoring device can automatically quantify an REI that compares well to the AHI scored manually on a concurrent PSG. Such a device may prove useful to assess SDB in high risk populations with self-administered testing in ambulatory settings such as the home.

Keywords Automated detection · OSA · Sleep apnea · SDB $\cdot$ Sleep-disordered breathing $\cdot$ Apnea $\cdot$ hypopnea . Nasal pressure $\cdot$ Portable monitoring $\cdot$ RUSleeping

\section{Introduction}

Sleep-disordered breathing (SDB) is a common disorder with important clinical consequences for affected individuals. Obstructive sleep apnea (OSA), a common form of $\mathrm{SDB}$, is highly prevalent in adults with approximately one in five adults having at least mild OSA and one in 15 adults having OSA with moderate severity or worse [1]. OSA is characterized by repetitive collapse of the pharyngeal airway during sleep, yielding hypoxia, hypercapnia, and arousal from sleep to reestablish airway patency [2]. The associated consequences include daytime sleepiness [3], 
decreased cognitive performance, decreased quality of life [4], increased risk of automobile and industrial accidents [5, 6], and adverse cardiovascular sequelae [7-10]. Treatment of OSA leads to improvements in many of these adverse outcomes and may reduce healthcare costs [11-13]. Thus, diagnosis of this disorder is important.

Referral to a sleep laboratory for overnight polysomnography (PSG) is the most common diagnostic pathway for patients with suspected sleep apnea. This procedure includes continuous recordings of many physiologic data including airflow, chest/abdominal excursion, electroencephalography, electro-oculography, electromyography, electrocardiography, and oxyhemoglobin saturation [14]. Apneas and hypopneas are detected with the monitoring equipment during periods of sleep to generate an apnea-hypopnea index (AHI) representing the number of these events per hour of sleep. However, PSG in the sleep laboratory is expensive, cumbersome, and not readily available in many geographic areas due to a growing demand for the procedure [15].

Assessing SDB with a portable monitoring device based on a limited number of channels in an ambulatory setting such as the home could improve access to care and reduce costs. Self-administered testing by the patient following instruction at the sleep laboratory by trained staff is the preferred method for acquiring the necessary physiologic data with such a device. The American Sleep Disorders Association (ASDA) has classified diagnostic systems into four categories based on the testing environment, technician attendance, and number of parameters recorded [16]. Level $\mathrm{I}$ is reserved for technician-attended in-laboratory PSG. Portable monitoring ranges from level II (unattended, full polysomnography) to level IV (single channel such as simple pulse oximetry). ASDA level III is reserved for devices that monitor airflow, chest/abdominal excursion, heart/pulse rate, and oxyhemoglobin saturation.

The portable monitoring device investigated in this study is a single-channel ASDA Level IV device that monitors changes in nasal pressure (a surrogate measure of airflow) with a nasal cannula, pressure transducer, and recording unit that provides signal processing and data analysis. The device monitors changes in nasal pressure to detect respiratory events and calculate an index representing the number of these events per hour of the overnight recording. Since the device only requires placement of a nasal cannula and securing the recording unit to the patient's bed clothing, it should be well-suited for self-administered testing. Furthermore, since the device automatically calculates the outcome of the overnight test, it requires minimal effort by the sleep laboratory staff or physician to conduct the test other than instructing the patient in its proper use. The aim of this study was to validate the output from the single-channel device against the standard for SDB detection, attended polysomnography in a laboratory setting.

\section{Materials and methods}

\section{Participants}

A convenience sample of adult patients referred to the Sleep Center of Greater Pittsburgh clinical sleep laboratory with suspected OSA participated in this investigation. Inclusion criteria included an age between 18 and 80 years, ability to provide informed consent and follow instructions, and medically stable status. Exclusion criteria were acute respiratory failure, periodic limb movement index greater than 10 events per hour on the night of the study, supplemental oxygen therapy, presence of tracheostomy, major nasal deformity (e.g., deviated septum), acute sinusitis or otitis media, or unwillingness or inability to tolerate a sleep study. Informed consent was obtained from all participants after the protocol was approved by the Institutional Review Board.

\section{Protocol}

All participants underwent standard in-laboratory overnight polysomnography (PSG). Signals recorded included electroencephalogram (C4-A1, C3-A2, O2-A1, and O1-A2), electro-oculogram, submental and bilateral tibial electromyogram, electrocardiogram, airflow (nasal-oral thermistor and nasal pressure [PTAF2, Pro-Tech Services, Woodinville, WA, USA]), chest and abdominal excursion (piezo bands), oxyhemoglobin saturation, and body position. All PSG data were collected and stored using the Sandman digital PSG system (Nellcor Puritan Bennett (Melville) Ltd., Kanata, ON, Canada). Nasal pressure was concurrently recorded on both the PSG and the portable monitoring device (RUSleeping ${ }^{\mathrm{TM}}$ RTS, Respironics, Murrysville, PA, USA). This was accomplished using a single nasal cannula attached to the subject and a Y-connector to split the cannula tubing to two pressure transducers: one for the PSG and one within the portable monitoring device.

PSGs were all scored manually by the same registered technologist at the Sleep Center of Greater Pittsburgh, who was blinded to the portable monitoring device data. Sleep was staged according to standard criteria [17]. Arousals were defined according to the American Sleep Disorders Association (ASDA) guidelines [18]. Apneas and hypopneas were scored according to the American Academy of Sleep Medicine (AASM) guidelines for measurement in clinical research [19]. An apnea-hypopnea index (AHI) was then calculated based on the number of apneas plus hypopneas per hour of sleep. 
The portable monitoring device included a small recording unit to monitor the change in nasal pressure with respiration using a nasal cannula. Power was provided for the device with a single $1.5 \mathrm{~V}$ AAA battery. Prior to beginning each overnight study with the device, the technologist first verified that airflow was being detected using the bar graph on the device display (six out of the 12 bars are indicated when no breathing is detected; then the number of bars increases with inspiration and decreases with exhalation). The technologist reset the internal counter on the device to zero before lights out. The device then continuously monitored the subject's respiration to detect respiratory events (maximum recording time: $9 \mathrm{~h}$ ). Respiratory events were scored by the device when the peak-topeak nasal pressure waveform initially fell at least below $50 \%$ of the baseline value (mean peak-to-peak airflow of eight breaths preceding initial breath of potential respiratory event) and remained below at least $75 \%$ of the baseline value for a minimum of $10 \mathrm{~s}$. If the respiratory event elapsed time counter reached $4 \mathrm{~min}$, then the signal was considered "not valid" for that period, the elapsed time counter was automatically reset, the respiratory event was discarded, and that hour of the recording was marked to indicate that a "not valid" period occurred. The device displayed the total elapsed hours of the recording and the real-time respiratory event index (REI) on the liquid crystal display (LCD). The REI represents the number of apneas and hypopneas detected by the portable monitoring device per hour of elapsed time for the recording. If a given hour had three "not valid" periods (at least 12 min without valid airflow), then that hour of the recording was discarded, marked as "ERR" and did not count toward the respiratory event index. At the conclusion of each overnight study, the REI reported on the recording unit display was documented by the technologist for future data analysis. The display was also queried to determine the number of respiratory events for each hour of the study and to determine if any hours were marked as "ERR".

Data analysis

Sleep studies were considered acceptable for data analysis if none of the following rejection criteria occurred: (1) PSG total sleep time (TST) less than $2 \mathrm{~h},(2)$ portable monitoring device elapsed time less than $2 \mathrm{~h}$, or (3) poor quality PSG recording (defined as a substantial portion of the PSG not being interpretable for the scoring of sleep and respiratory events). The PSG was considered the standard for identifying and quantifying SDB. The portable monitoring device REI was compared to the AHI calculated from the PSG using the previously mentioned criteria.

The utility and accuracy of the portable monitoring device REI in detecting SDB was based on summary data and was evaluated in a number of ways. These included evaluations of concordance using linear regression and agreement using the method of Bland and Altman [20]. To assess the detection of varying degrees of SDB severity, we constructed receiver operating characteristic (ROC) curves [21] (Systat Software, SigmaPlot 10.0) using PSG AHI thresholds of 5,15 , and 30 corresponding to mild, moderate, and severe SDB, respectively.

All results are given as means \pm 1 standard deviation except when standard error is indicated. The differences for the Bland-Altman analyses are given as means (2 SD for limits of agreement). Statistical significance was considered to be present when $p<0.05$.

\section{Results}

We recruited and enrolled a total of 25 subjects with suspected OSA, who met prospective eligibility requirements for participation in this investigation. This was not a consecutive series of subjects, but a convenience sample of willing participants who met enrollment criteria when staff and beds were available to conduct the investigation. No PSGs or portable device studies met our criteria for data failure, and the results from all 25 enrolled subjects (16 female) are included in the data analysis. Five subjects had $1 \mathrm{~h}$, one had $2 \mathrm{~h}$, and one had $4 \mathrm{~h}$ marked as "ERR" (representing hours with at least 12 min of "not valid" airflow) by the portable device. Table 1 provides descriptive data and the distribution of SDB severity (based on PSG) for all participants. The PSG mean recording time was $391.2 \pm 41.3 \mathrm{~min}$ and the PSG mean total sleep time was 319.0 $\pm 83.2 .0 \mathrm{~min}$. The portable monitoring device

Table 1 Descriptive data for all subjects and based on SDB severity using PSG AHI

\begin{tabular}{lrlll}
\hline & $N$ & Gender & Age (years) & BMI $\left(\mathrm{kg} \mathrm{m}{ }^{-2}\right)$ \\
\hline All Subjects & 25 & $9 \mathrm{M}, 16 \mathrm{~F}$ & $42.4 \pm 12.9$ & $31.0 \pm 7.4$ \\
No SDB AHI $<5 \mathrm{~h}^{-1}$ & 7 & $1 \mathrm{M}, 6 \mathrm{~F}$ & $27.7 \pm 6.1$ & $26.6 \pm 4.3$ \\
Mild SDB $\left(5 \geq \mathrm{AHI}<15 \mathrm{~h}^{-1}\right)$ & 6 & $1 \mathrm{M}, 5 \mathrm{~F}$ & $41.1 \pm 6.9$ & $30.0 \pm 7.0$ \\
$\begin{array}{l}\text { Moderate SDB }(15 \geq \mathrm{AHI} \\
\left.\quad<30 \mathrm{~h}^{-1}\right)\end{array}$ & 7 & $3 \mathrm{M}, 4 \mathrm{~F}$ & $52.5 \pm 10.9$ & $33.4 \pm 6.7$ \\
Severe SDB $\left(\mathrm{AHI} \geq 30 \mathrm{~h}^{-1}\right)$ & 5 & $4 \mathrm{M}, 1 \mathrm{~F}$ & $50.8 \pm 8.0$ & $37.4 \pm 8.6$ \\
\hline
\end{tabular}


recorded a mean of $6.7 \pm 1.3 \mathrm{~h}$ with valid airflow per subject. The mean PSG AHI was $20.6 \pm 26.8$ events per hour while the mean REI calculated by the portable monitoring device was $18.1 \pm 11.5$ events per hour. There was a significant correlation between the PSG AHI and portable monitoring device REI $(R=0.73, p<0.0001$, Fig. 1a). Agreement was assessed using the Bland-Altman plot as shown in Fig. 1b. There was good agreement across a wide range of SDB severity except where the REI underestimated the AHI by 87.7 events per hour in one subject with an AHI of 133.5 events per hour.

We constructed ROC curves (see Figs. 2, 3 and 4) to assess the sensitivity and specificity of the portable monitoring device using a range of PSG AHI threshold values (5, 15 and 30 events per hour) to differentiate normal cases from those with SDB. The area under the curve (AUC) was 0.94 (SE $0.05, p<0.001,95 \%$ CI $0.84-1.0$ ), 0.88 (SE 0.07, $p<0.001,95 \%$ CI 0.74-1.0), and 0.91 (SE $0.06, p<0.001,95 \%$ CI $0.79-1.0$ ), respectively. Table 2
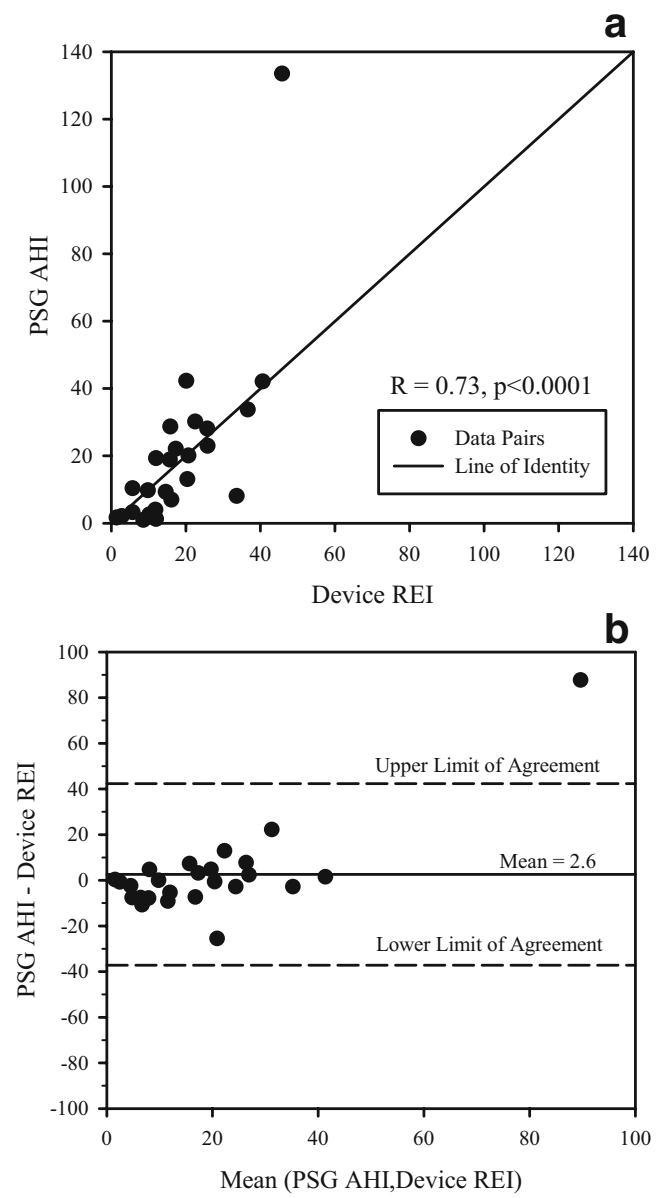

Fig. 1 In-laboratory comparisons are shown. a Scatter plot of PSG AHI vs. Device REI. b Bland-Altman plot of PSG AHI vs. Device REI. Agreement was good and the difference on the Bland-Altman plot remained relatively constant for increasing severity of SDB except for one subject with a PSG AHI of 133.5 events per hour

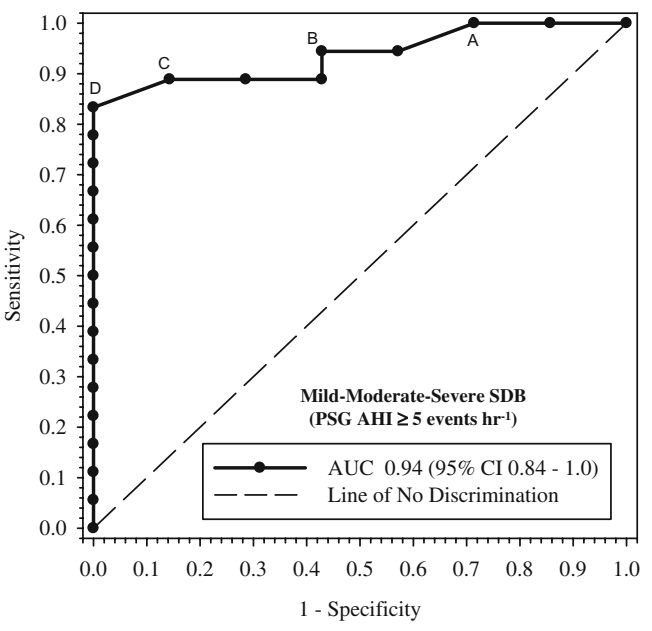

Fig. 2 ROC curve for discriminating subjects with SDB (PSG AHI $\geq 5$ events per hour) from those without SDB using the portable monitoring device REI (solid line). The AUC is 0.94 (SE 0.05, $p<$ $0.001,95 \%$ CI $0.84-1.0$ ). The ROC curve is constructed using increasing cutoffs for the REI to detect SDB. Four of these cutoffs are marked and represent (A: REI 4.3, B: REI 9.2, $C$ : REI 11.9, and $D$ : REI 13.3) Definition of abbreviations: ROC receiver operating characteristic, $S D B$ sleep-disordered breathing, $P S G$ polysomnogram, $A H I$ apnea-hypopnea index, $R E I$ respiratory event index, $A U C$ area under curve

provides the sensitivity, specificity, and corresponding likelihood ratios for the marked REI cutoffs on Figs. 2, 3, and 4.

\section{Discussion}

In this investigation, we assessed the diagnostic accuracy of a simple portable device to detect sleep-related respiratory

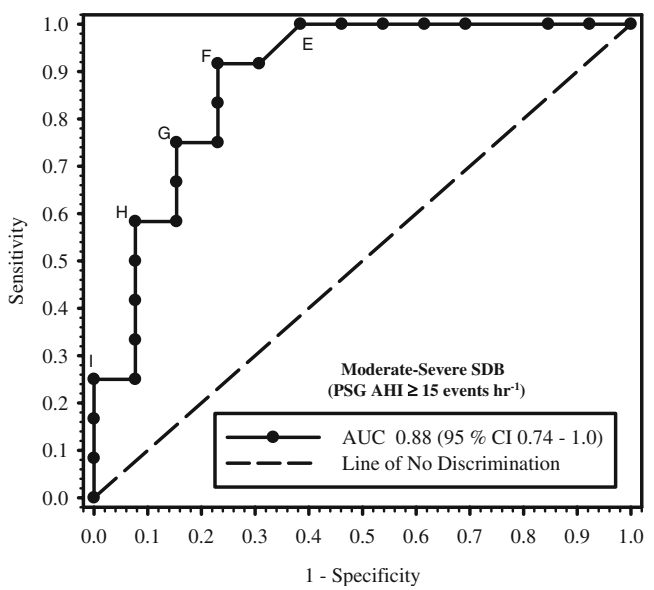

Fig. 3 ROC curve for discriminating subjects with moderate-severe SDB (PSG AHI $\geq 15$ events $h^{-1}$ ) from those without moderate-severe SDB using the portable monitoring device REI (solid line). The AUC is 0.88 ( $\mathrm{SE} 0.07, p<0.001,95 \%$ CI $0.74-1.0$ ). The ROC curve is constructed using increasing cutoffs for the REI to detect SDB. Five of these cutoffs are marked and represent $(E$ : REI 11.9; $F$ : REI 15.2; $G$ : REI 16.7; $H$ : REI 20.6, and $I$ : REI 35.1) 


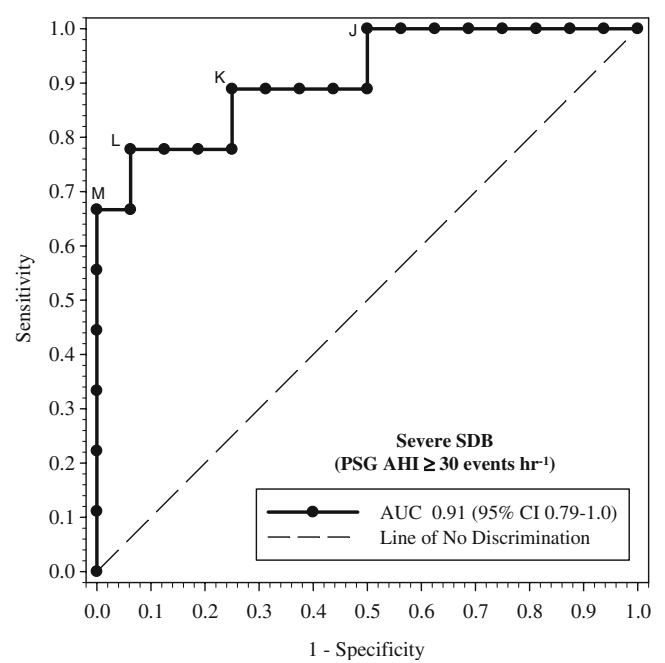

Fig. 4 ROC curve for discriminating subjects with severe SDB (PSG $\mathrm{AHI} \geq 30$ events per hour) from those without severe SDB using the portable monitoring device REI (solid line). The AUC is 0.91 (SE $0.06, p<0.001,95 \%$ CI $0.79-1.0$ ). The ROC curve is constructed using increasing cutoffs for the REI to detect SDB. Four of these cutoffs are marked and represent $(J$ : REI 7.6, $K$ : REI 16.0, $L$ : REI 22.6, and $M$ : REI 28.4)

events with concurrent PSG in the sleep laboratory in a population with suspected SDB. This device monitors a single channel (nasal pressure) to detect respiratory events and continuously reports a real-time REI based on the number of respiratory events per hour of recording time. The portable device REI agreed with the degree of SDB determined by the PSG AHI (see Fig. 1). The REI area under the ROC curve ranged from 0.88 to 0.94 for all levels of SDB (see Figs. 2-4). Technical failures (i.e. less than $2 \mathrm{~h}$ of reliable data during the recording time) did not occur in the study and hours with more than 12 min of "not valid" airflow were uncommon. These data extend previous findings for this portable monitoring device [22-24].

These data also suggest this portable device can be used to increase the pretest odds for SDB (PSG AHI $\geq 5$ events per hour) by $620 \%$ with a positive test (REI $>11.9$ events per hour) while reducing the posttest odds to $13 \%$ of the pretest odds for a negative test (REI $\leq 11.9$ events per hour). Thus, the device offers clinical utility as a simple method to assess the presence of SDB (at any level) in high-risk populations such as patients with diabetes, refractory hypertension, and other cardiovascular disorders. The device provides similar results to detect moderate-severe SDB and may prove useful in clinical applications such as screening patients prior to surgery or to assess efficacy of mandibular advancement devices.

The portable device we investigated offers several potential advantages over other portable monitoring systems. First, the device consists of only a small recording unit that can be easily clipped onto the subject's bedclothes and requires only a single sensor attachment (nasal cannula) to acquire the necessary data. This makes the device well suited for self-administered testing in an ambulatory environment such as the home where numerous sensors can impair the reliability for collecting data in remote settings. Second, the device automatically calculates a realtime REI without downloading data to a computer. This reduces the sleep laboratory resources required to dispense the devices, educate patients on proper use, and determine the REI. Third, the disposable supplies needed to conduct a study are limited to a single nasal cannula. This offers a

Table 2 Sensitivity, specificity, and likelihood ratios corresponding to REI cutoffs marked on the ROC curve figures

\begin{tabular}{|c|c|c|c|c|c|c|c|c|}
\hline SDB severity & Figure marker & REI $>$ & Sensitivity & $95 \% \mathrm{CI}$ & Specificity & $95 \% \mathrm{CI}$ & $\mathrm{LR}+$ & $\mathrm{LR}-$ \\
\hline \multirow[t]{4}{*}{ PSG $\mathrm{AHI} \geq 5$} & A & 4.3 & 1.0 & $0.81-1.0$ & 0.29 & $0.04-0.71$ & 1.4 & 0.00 \\
\hline & B & 9.2 & 0.94 & $0.73-1.0$ & 0.57 & $0.18-0.90$ & 2.2 & 0.10 \\
\hline & $\mathrm{C}$ & 11.9 & 0.89 & $0.65-0.99$ & 0.86 & $0.42-1.0$ & 6.2 & 0.13 \\
\hline & $\mathrm{D}$ & 13.3 & 0.83 & $0.59-0.96$ & 1.00 & $0.59-1.0$ & $\infty$ & 0.17 \\
\hline \multirow[t]{5}{*}{ PSG $\mathrm{AHI} \geq 15$} & $\mathrm{E}$ & 11.9 & 1.0 & $0.74-1.0$ & 0.62 & $0.32-0.86$ & 2.6 & 0.00 \\
\hline & $\mathrm{F}$ & 15.2 & 0.92 & $0.62-1.0$ & 0.77 & $0.46-0.95$ & 4.0 & 0.11 \\
\hline & G & 16.7 & 0.75 & $0.43-0.95$ & 0.85 & $0.55-0.98$ & 4.9 & 0.30 \\
\hline & $\mathrm{H}$ & 20.6 & 0.58 & $0.28-0.85$ & 0.92 & $0.64-1.0$ & 7.6 & 0.45 \\
\hline & I & 35.1 & 0.25 & $0.05-0.57$ & 1.00 & $0.75-1.0$ & $\infty$ & 0.75 \\
\hline \multirow[t]{4}{*}{$\mathrm{PSG} \quad \mathrm{AHI} \geq 30$} & $\mathrm{~J}$ & 7.6 & 1.0 & $0.66-1.0$ & 0.50 & $0.25-0.75$ & 2.0 & 0.00 \\
\hline & $\mathrm{K}$ & 16.0 & 0.89 & $0.52-1.0$ & 0.75 & $0.48-0.93$ & 3.6 & 0.15 \\
\hline & $\mathrm{L}$ & 22.6 & 0.78 & $0.40-0.97$ & 0.94 & $0.70-1.0$ & 12.4 & 0.23 \\
\hline & M & 28.4 & 0.67 & $0.30-0.93$ & 1.00 & $0.79-1.0$ & $\infty$ & 0.33 \\
\hline
\end{tabular}

Abbreviations: $R O C$ receiver operating characteristic, SDB sleep-disordered breathing, $P S G$ polysomnogram, $A H I$ apnea-hypopnea index, $R E I$ respiratory event index, $A U C$ area under curve, $C I$ confidence interval, $L R+$ likelihood Ratio for a positive $(+)$ test, $L R$ likelihood ratio for a negative (-) test. 
cost advantage over other systems, which require expensive single patient use supplies to conduct a study.

Our investigation and the device also have a number of limitations. First, the study was limited to a small number of eligible participants who were willing to participate in a research study at only one sleep center. The proportion of females in our study was also high for a sleep lab population. Thus, these results may not extend to other populations. Second, the data were acquired on a single night in a sleep laboratory and did not include an additional night in an ambulatory environment such as the home. However, comparisons on different nights and different testing environments (lab vs. home) are not ideal. Third, the device detects sleep disordered breathing with a single nasal pressure channel, so some events may be difficult to detect. Fourth, the raw nasal pressure data were not available for review or for editing the scoring of events. Thus, our data were automatically calculated by the portable device and were unedited. Fifth, the portable device cannot calculate an REI specific to body position nor can it differentiate apneas from hypopneas or central from obstructive apneas nor can it exclude respiratory events during periods of irregular breathing patterns during wake. These limitations may prove unsatisfactory in determining a differential diagnosis in some patients.

In conclusion, this study indicates that the RUSleeping ${ }^{\mathrm{TM}}$ RTS portable monitoring device can accurately assess the presence and severity of SDB compared to concurrent polysomnography in the sleep laboratory, with a low failure rate for single use and minimal technician time compared to PSG. Due to the estimated prevalence of SDB in the general population that can be substantially higher in high-risk groups, access to timely diagnosis and treatment is important. As sleep physicians and technologists become more familiar with ambulatory monitoring technology, portable devices will play a greater role in the management of this disorder. As a field, we must evolve our sleep disorder programs to not only focus on the next patient that walks through the clinic door for evaluation, but also the leadership to manage sleep disorders for all patients within our communities. For this to become a reality, the future of sleep medicine will likely include a more diverse diagnostic armamentarium consisting of sleep laboratory-based polysomnography, limited channel cardiopulmonary studies, AND even single-channel portable monitoring in the home. Otherwise, it is just one-size-fits-all: in-lab polysomnography to diagnose SDB and titrate CPAP.

Acknowledgments The authors would like to thank the clinical staff of the Greater Pittsburgh Sleep Center for the data collection/scoring and Richard Chiacchierini, PhD of R.P. Chiacchierini \& Associates for the data analysis.

Disclosure This study was funded by Respironics, Inc., Murrysville, PA. Dr. Grover has received grant-research support from Respironics,
Inc. SD Pittman is an employee of Respironics, Inc, and has no financial interest in a sleep laboratory.

\section{References}

1. Young T, Skatrud J, Peppard PE (2004) Risk factors for obstructive sleep apnea in adults. JAMA 291(16):2013-2016

2. Remmers JE, deGroot WJ, Sauerland EK, Anch AM (1978) Pathogenesis of upper airway occlusion during sleep. J Appl Physiol 44(6):931-938

3. Roehrs T, Conway W, Wittig R, Zorick F, Sicklesteel J, Roth T (1985) Sleep-wake complaints in patients with sleeprelated respiratory disturbances. Am Rev Respir Dis 132(3): 520-523

4. Flemons WW, Tsai W (1997) Quality of life consequences of sleep-disordered breathing. J Allergy Clin Immunol 99(2):S750 S756

5. Findley LJ, Unverzagt ME, Suratt PM (1988) Automobile accidents involving patients with obstructive sleep apnea. Am Rev Respir Dis 138(2):337-340

6. Teran-Santos J, Jimenez-Gomez A, Cordero-Guevara J (1999) The association between sleep apnea and the risk of traffic accidents. Cooperative Group Burgos-Santander. N Engl J Med 340(11):847-851

7. Dyken ME, Somers VK, Yamada T, Ren ZY, Zimmerman MB (1996) Investigating the relationship between stroke and obstructive sleep apnea. Stroke 27(3):401-407

8. Hung J, Whitford EG, Parsons RW, Hillman DR (1990) Association of sleep apnoea with myocardial infarction in men. Lancet 336(8710):261-264

9. Lavie P, Herer P, Hoffstein V (2000) Obstructive sleep apnoea syndrome as a risk factor for hypertension: population study. BMJ 320(7233):479-482

10. Peppard PE, Young T, Palta M, Skatrud J (2000) Prospective study of the association between sleep-disordered breathing and hypertension. N Engl J Med 342(19):1378-1384

11. Kribbs NB, Pack AI, Kline LR et al (1993) Effects of one night without nasal CPAP treatment on sleep and sleepiness in patients with obstructive sleep apnea. Am Rev Respir Dis 147(5):11621168

12. Kryger MH, Roos L, Delaive K, Walld R, Horrocks J (1996) Utilization of health care services in patients with severe obstructive sleep apnea. Sleep 19(9 Suppl):S111-116

13. Rodenstein DO (2000) Sleep apnoea syndrome: the health economics point of view. Monaldi Arch Chest Dis 55(5):404410

14. Polysomnography Task Force, American Sleep Disorders Association Standards of Practice Committee (1997) Practice parameters for the indications for polysomnography and related procedures. Sleep .20(6):406-422

15. Pack AI, Gurubhagavatula I (1999) Economic implications of the diagnosis of obstructive sleep apnea. Ann Intern Med 130(6):533534

16. Ferber R, Millman R, Coppola M et al (1994) Portable recording in the assessment of obstructive sleep apnea. ASDA standards of practice. Sleep 17(4):378-392

17. Rechtschaffen A, Kales A (1968) A manual of standardized terminology, techniques, and scoring system for sleep stages of human subjects. Brain Information Service, Los Angeles

18. American Sleep Disorders Association. (1992) (1992) EEG arousals: scoring rules and examples: a preliminary report from the Sleep Disorders Atlas Task Force of the American Sleep Disorders Association. Sleep 15(2):173-184 
19. American Academy of Sleep Medicine Task Force (1999) (1999) Sleep-related breathing disorders in adults: recommendations for syndrome definition and measurement techniques in clinical research. The report of an American Academy of Sleep Medicine Task Force. Sleep 22(5):667-689

20. Bland JM, Altman DG (1986) Statistical methods for assessing agreement between two methods of clinical measurement. Lancet 1(8476):307-310

21. Hanley JA, McNeil BJ (1982) The meaning and use of the area under a receiver operating characteristic (ROC) curve. Radiology 143(1):29-36
22. Gorny SW, Allen RP, Krausman DT (2000) Evaluation of an unattended monitoring system for automated detection of sleep apnea. Sleep 23(Suppl 2):A369

23. Gorny SW, Spiro JR, Phillips B, Allen RP, Krausman DT (2001) Initial findings from a multi-site evaluation of an unattended monitoring system for automation detection of sleep-disordered breathing events. Sleep 24(Suppl):A387

24. Spiro JR, Gorny SW, Allen RP, Krausman DT (2002) Pilot evaluation of an ambulatory airflow pressure monitor for immediate identification of sleep-disordered breathing events. Sleep 25(Suppl):A275 